\title{
CD133 Antigen Peptide-pulsed Autologous Dendritic Cell Vaccine
}

National Cancer Institute

\section{Source}

National Cancer Institute. CD133 Antigen Peptide-pulsed Autologous Dendritic Cell

Vaccine. NCl Thesaurus. Code C113794.

A cell-based cancer vaccine comprised of autologous dendritic cells (DCs) pulsed with human leukocyte antigen (HLA)-A2-restricted peptides derived from the CD133 antigen, with potential antineoplastic activity. Upon intradermal administration, the CD133 antigen peptide-pulsed autologous DC vaccine may stimulate an anti-tumoral cytotoxic Tlymphocyte (CTL) response against CD133-expressing tumor cells, resulting in tumor cell lysis. CD133, a cancer stem cell marker, is expressed on hematopoietic stem and progenitor cells and overexpressed on many types of cancer cells; it is associated with resistance to chemotherapy and increased cancer survival. HLA-A2 is an MHC class I molecule that presents antigenic peptides to CD8+ T-cells. Epitope design that is restricted to those epitopes that bind most efficiently to HLA-A2 may improve antigenic peptide immunogenicity. 